\title{
Universal features in lifetime distribution of clusters in hydrogen bonding liquids
}

\author{
Ivo Jukic ${ }^{\dagger \dagger}$, Bernarda Lovrinčević ${ }^{\ddagger *}$, Martina Požar ${ }^{\ddagger}$, and Aurélien Perera ${ }^{\dagger \dagger}$
}

May 10, 2021

${ }^{\dagger}$ Laboratoire de Physique Théorique de la Matière Condensée (UMR CNRS

7600), Sorbonne Université, 4 Place Jussieu, F75252, Paris cedex 05, France.

${ }^{\ddagger}$ Department of Physics, Faculty of Sciences, University of Split, Ruđera

Boškovića 37, 21000, Split, Croatia.

\begin{abstract}
Hydrogen bonding liquids, typically water and alcohols, are known to form labile structures (network, chains, etc...), hence the lifetime of such structures is an important microscopic parameter, which can be calculated in computer simulations. Since these cluster entities are mostly statistical in nature, one would expect that, in the short time regime, their lifetime distribution would be a broad Gaussian-like function of time, with a single maximum representing their mean lifetime, and weakly dependent on criteria such as the bonding distance and angle, much similarly to nonhydrogen bonding simple liquids, while the long time part is known to have some power law dependence. Unexpectedly, all the hydrogen bonding liquids studied herein, namely water and alcohols, display highly hierarchic three types of specific lifetimes, in the sub-picosecond range 0-0.5ps The dominant lifetime very strongly depends on the bonding distance criterion and is related to hydrogen bonded pairs. This mode is absent in non-Hbonding simple liquids. The secondary and tertiary mean lifetimes are related to clusters, and are nearly independent on the bonding criterion. Of these two lifetimes, only the first one can be related to that of simple liquids, which poses the question of the nature of the third life time. The study of acohols reveals that this 3rd lifetime is related to the topology of H-bonded clusters, and that its distribution may be also affected by the alkyl tail surrouding ?bath?. This study reveals that hydrogen bonding liquids have a universal hierarchy of hydrogen bonding lifetimes with a timescale regularity across very different types, and which depend on the topology of the cluster structures
\end{abstract}

\footnotetext{
*bernarda@pmfst.hr

${ }^{\dagger}$ corresponding author (aup@lptmc.jussieu.fr)
} 


\section{Introduction}

Labile structures in associated liquids and mixtures pose the problem of the role of kinetics of said structures, and its influence on the thermophysical and dynamical properties of these systems [1, 2]. Such structures play an important role in soft matter, as for example with micelles and lamellae [3, 4, 5], and more particularly in biology, wherein the labile character of various functional molecular entities, such as enzymes for example, wears a fundamental operational nature [6, 7, 8]. One might even postulate that biological systems have been built by the increasing role played by such labile structures in the early evolution of primitive biochemical systems [9, 10]. Based on these premises, it is important to better understand the interplay between labile nature of molecular assemblies and the role of kinetics in their dynamics [1, 6, 7, 8,

A first step in that direction would be analyzing the life time distribution of the hydrogen bonding process which is at the root of molecular association 11, 12. Since H-bonding is essentially a quantum mechanical process, it is affected by various intra molecular motions, and in turn it affects inter molecular motions. It is not clear if the various experimental techniques, which allow to probe the frequencies associated with these motions, can unambiguously answer the question posed above [13, 14, 15, 16. Since this is a many body quantum mechanical phenomenon, it is not even clear if approximate theories can provide an alternative approach to this question. On the other hand, computer simulation provide a direct access to the statistics of molecular motions, and are able to answer this question. One could even answer this question at the level of classical physics, where the hydrogen bond is modeled by the Coulomb pairing of opposite charges [17, 18, and whose pertinence has been amply proven by more than fifty years of computer simulations and force field development.

In fact, the question posed above has been already partly answered by Luzar and Chandler in their 1996 Nature paper [19]. In this paper, the authors focus essentially in the long time kinetics of H-bonding in water, beyond the initial $0.5 \mathrm{ps}$ which they mention as the transient regime. This choice is amenable to a theoretical approach of the H-bonding kinetics, which is shown to be non-exponential, and further supported by classical computer simulations, but it does not explain the origin of the transient behaviour observed at short times. The present work aims at revealing surprising repeatability of this transient part across several H-bonding liquids. It is initially motivated by the fact that $\mathrm{H}$-bonding liquids other than water, such as alcohols and amines, are known to form short chain-like clusters, both from scattering experiments [20, 21, 22, 23, 24, 25, 26, 27, 28, 29, 30, 31, 11, 32, spectroscopy investigations [33, 34, 35, 36, 37] and computer simulations [38, 39, 40, 41, 42, 43, 44]. Exploring this transient regime, essentially in the sub 0.5 ps region, we have uncovered a universal dynamical behaviour common to all H-bonding liquids, which is unexpected in this time domain, where the differences in the molecular interactions play an important role. Indeed, such universality would be more expected in the long time kinetics regime, where common features of the association process are likely to settle [2, 45]. 
In order to better appreciate the results presented herein, it is useful to picture the $\mathrm{H}$-bonding process as essentially a random process at short times and distances, governed by the random molecular encounters. Intuitively, one would expect a broad distribution of $\mathrm{H}$-bonding life times, as function of both time and $\mathrm{H}$-bonding distance, centered around some mean representative life time, which could be subsequently searched in the various experimental techniques investigating the relaxation processes. What we uncover here, is that while the distribution of $\mathrm{H}$-bonding distances are indeed as hypothesized, the life time distributions exhibit several specific times, which vary from 1 at very short distances to 3 at larger ones, where the distances are picked around the main peak of the oxygen-oxygen distribution function, typically in the range $2.5 \AA$ to $3.5 \AA$, this latter value being often used in the literature [46, 47, 48, 49], as in the Luzar paper mentioned above [19. These specific times, and related distribution, appear as common to all three associated liquids we have investigated herein, namely water, alcohols and amine, and across different force field representation. In contrast, the long time kinetics show specificities related to each type of liquid. Although we have used classical force field, this universality strongly suggests that these times should be a real feature of the associated liquids. We suggest that these specific times correspond to three types of molecular associations: dimer, linear chain-like clusters and other types of clusters. This finding suggests that self-assembled labile structures have typical life times related to their differences in microscopic topology. In that, it helps understand how such structures, when complexified by appropriate molecular entities, could acquire an important role in the pre-biotic phenomena. More importantly, it shifts the interest to such structures, from the usual long time kinetics approach, where kinetic constants play an important role, to short times and distance, in the range of which new self assembled objects appear, and could possibly play the role of new molecular species in a very restricted spatio-temporal region.

\section{Theoretical, models and simulation details}

We would like to first stress that the present manuscript deals with the H-bond lifetime distribution itself, and not the time auto-correlation of it, which has been the subject of previous works by other authors [19, 50, 51, 52, [53]. It is therefore important to clarify the differences in the theoretical backgrounds. The hydrogen bond is a real property of many associated liquids in many contexts. However, for calculational purposes, it is necessary do define the two following parameters, the bonding distance $r_{c}$ between the two donor/acceptor atoms, and the corresponding bonding angle $\theta_{c}$. In computer simulations, two molecules $i$ and $j$ are considered as H-bonded, when the distance $r_{i j}$ between the corresponding donor/acceptor atoms $A_{i}$ and $B_{j}$ obeys $r_{i j} \leq r_{c}$, and the angle $\theta_{i j}=A_{i} \widehat{H B} B_{j}$ obeys $\left|\theta_{i j}\right|<\theta_{c}$. The $A_{i}$ and $B_{j}$ atoms are typically oxygen atoms, such as in water, but they can also refer to nitrogen atoms, such as for 1-propylamine considered in this work. Below, we will refer as $\mathcal{C}$ the ensemble of atoms which verify both criteria. For each pair of molecules in this time inter- 
val, there are 2 characteristic times: that $t_{i j}$ when they first bond and $\tau_{i j}$ when they break apart for the first time. For this pair, we define a time dependant random variable $H_{i j}(t)$, such that

$$
H_{i j}(t)=H\left(t-t_{i j}\right) H\left(\tau_{i j}-t\right)
$$

where $H(t)$ is the Heaviside function, and $H_{i j}(t)=1$ for $t_{i j}<t<\tau_{i j}$ and zero elsewhere.

We believe that this is the first proper definition of the function $h(t)$ introduced in the past literature [19, 50, 51, 52, 53]. From this random variable one can measure several statistical averages and correlation, and in particular the auto-correlation function $c(t)=<h(0) h(t)>$ which has been studied in the past.

In the present work, we focus on the lifetime distribution itself, and the appropriate random variable is $h_{i j}(t)$ can defined from $H_{i j}(t)$. We first take the derivative $d H_{i j}(t) / d t=\delta\left(t-t_{i j}\right)-\delta\left(t-\tau_{i j}\right)$, and remove the origin part. Then we define $h_{i j}(t)$ as gauge variable (because of the derivative/integration operations) related to $H_{i j}(t)$ through

$$
h_{i j}(t)=\int d t \delta\left(t-\tau_{i j}\right)
$$

which is 1 when the Hbond breaks at time $\tau_{i j}$ and zero elsewhere.

This variable is therefore adapted to built the lifetime histogram, and the associated lifetime distribution defined as

$$
L(t)=\frac{1}{T_{0} L_{0}} \sum_{i j \in \mathcal{C}} h_{i j}(t)
$$

where the normalisation factor $L_{0}$ is defined as

$$
L_{0}=\frac{1}{T_{0}} \int_{0}^{T_{0}} d t\left[\sum_{i j \in \mathcal{C}} h_{i j}(t)\right]
$$

It is easily verified that $L(t)$ is a probability distribution which verifies

$$
\int_{0}^{T_{0}} d t L(t)=1
$$

Eqs. (34) provide a direct computational indication as how to evaluate $L(t)$ in a given computer simulation. An average over all possible time origin is implict. The auxiliary Gromacs program gmx hbond module with the -life option, allows to compute $L(t)$. We have also checked through our own code that it was consistent with the definitions given above. It should be noted that one can end the H-bonding as soon as two bonded atoms part away according to the chosen criterion. This is the strict definition, which has been adopted in this work, but also in Gromacs. However, in reality, a broken bond could be reformed quickly, and perhaps some margin should be allowed for rebinding, which could 
be added to the ensemble of criterion in $\mathcal{C}$. This could be conveniently introduced by replacing the Dirac delta in Eq. $(2)$ by a Gaussian function, which we will consider in another context.

This study focuses on the typical hydrogen bonding liquids, which are water and alcohols, which are both based on the $\mathrm{OH}$ group. Mono-ols such as methanol, ethanol and propanol have been studied. The SPC/E [54 and TIP4P_2005 [55] models were used to simulate water. Alcohols were modeled with the OPLS-UA [56] and TRaPPE-UA [57] forcefields.

The program package Gromacs, version 2018.1 [58, 59] was used for all molecular dynamics simulations. The simulation protocol has been the same for all neat systems. The initial configurations of $\mathrm{N}$ molecules were generated by random molecular positioning with the program Packmol [60, which were then energy minimized. The values for $\mathrm{N}$ considered here are typically $\mathrm{N}=1000$ or $\mathrm{N}=2048$. The systems were then equilibrated in the isobaric constant NpT ensemble for at least $5 \mathrm{~ns}$, followed by a production run of at least $5 \mathrm{~ns}$. Finally, an additional production run of 300 ps was performed to gather sufficient data for the analysis of dynamic quantities (every configuration was sampled).

The integration algorithm of choice was the leap-frog [61] and the time step was 2 fs. The electrostatics were handled with the PME method 62 and the constraints with the LINCS algorithm [63]. The short-range interactions were calculated within the $1.5 \mathrm{~nm}$ cut-off radius. Each neat liquid was simulated at ambient conditions. Temperature was maintained at $\mathrm{T}=300 \mathrm{~K}$ using the Nose-Hoover 64, 65 thermostat, while pressure was kept at $\mathrm{p}=1$ bar with the Parrinello-Rahman barostat 66, 67. The temperature algorithms had a time constant of $0.2 \mathrm{ps}$, while the pressure algorithm was set at $2 \mathrm{ps}$.

The lifetime calculation is part of the post simulation analysis Gromacs package (gmx H-bond module) . It follows the usual process of monitoring the lifetime of pairs of $\mathrm{H}$-bonded $\mathrm{O}$ atoms across different water molecules. The $\mathrm{O}-\mathrm{O} \mathrm{H}$-bonding distance $r_{c}$ and the $\mathrm{O}-\mathrm{H}-\mathrm{O}$ angle are input parameters. In this study, while we vary the bonding distance, the angle is maintained to the accepted value of $180^{\circ} \pm 30$. We have verified that there is much less dependence on the angular bias than the distance $r_{c}$.

\section{Results}

Usually, H-bonding is discussed in terms of the spatial distribution, through pair correlation functions and their spatial representations, as proven by their overwhelming representation in the literature, as compared with their temporal

one. Therefore, the finding of a corresponding temporal universality can be considered as an important feature, which should help better understand the role of labile structures. 


\subsection{Static structural properties of $\mathbf{H}$-bonding}

The universality in kinetics claimed in this work is supported by the static properties, in particular by the structural properties. Fig.1 shows the various oxygen-oxygen pair correlation functions $g_{O O}(r)$ obtained from computer simulations of the SPC/E water model, and first two OPLS alcohol models. The first peaks, shown in the main panel, demonstrate that the contact pairing is dominated by the oxygen atom size as well as the strong H-bonding pairing induced by the O-H-O Coulomb association. It is important to note that these first peaks are relatively robust across models, such that the distributions of bonding distances do not vary much across models. This is further enforced by the striking resemblance of distribution times across models, as shown below and in the SI material.

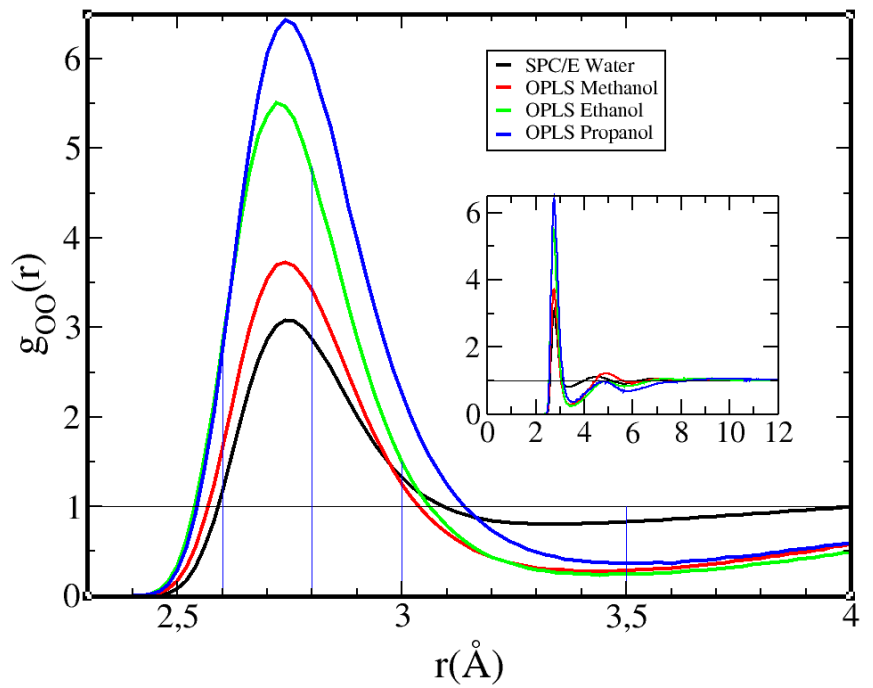

Figure 1: Oxygen-oxygen distribution functions $g_{O O}(r)$ for water and first alcohols. The vertical blue lines represent a sample of the H-bonding distances used in this work. The inset represent a wider range of these functions.

We turn to the H-bond donor-acceptor distance distributions, which is shown in Fig.2, for the same models as in Fig.1. We immediately note the expected feature discussed in the Introduction, namely that the distribution is indeed a broad one, centered around a distance which corresponds to the central peak in Fig.1, around 2.7 . It confirms that the underlying bonding is essentially a random phenomenon with no particular spatial specificity. This finding is somewhat in contrast with what snapshots of the cluster analysis reveal, where such clusters in water appear in various patterns, while for alcohols it has been proven that chains and loops are predominant.

The curves in Fig.2 tend to indicate that donor-acceptor distribution is essentially dominated by the dimers. Differences in clustering are found in the 


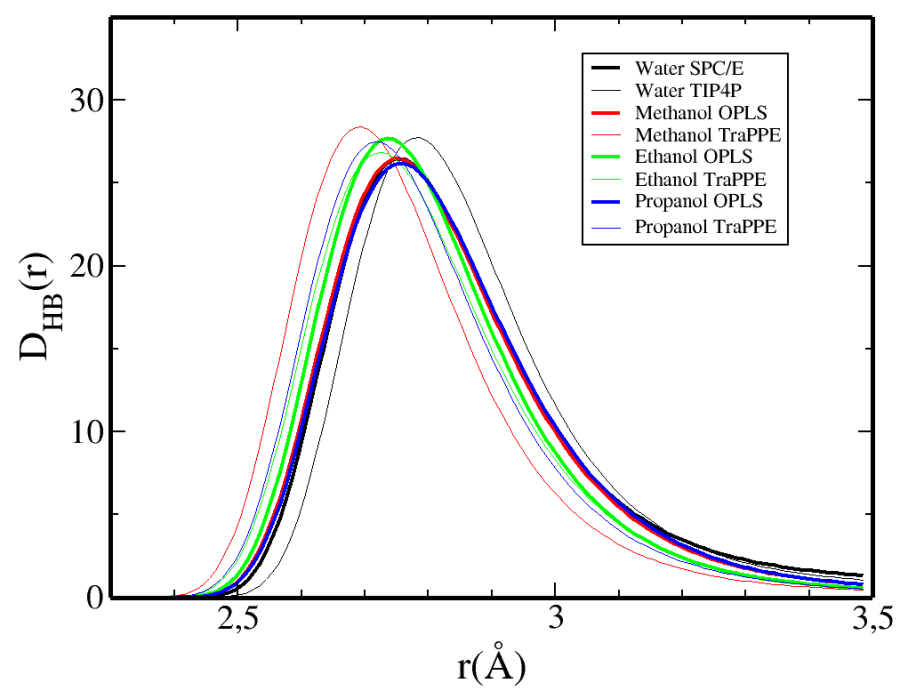

Figure 2: H-bond spatial distribution function $D_{H B}(r)$ for the different models studied herein.

larger distance in $g_{O O}(r)$, which is shown in Fig.1. Indeed, it is seen in the inset of Fig. 1 that the $g_{O O}(r)$ of the alcohols appears more depleted in the distance range $4-6 \AA$, which indicates that chain clusters are predominant in alcohols [40, 41, 32]. A direct consequence of this difference in clustering is the fact that the scattering intensities of alcohols differ markedly from that of water, since they exhibit a pre-peak. [22, 23, 24, 25, 28, 32]. Perhaps the next most striking feature in Fig.2 is the near exact superposition of all the curves, indicating that the H-bonding between oxygen atoms is near invariant across molecular species. Small variations are attributable to parameter differences in the underlying classical force field, such as the partial charges and atom Lennard-Jones parameters.

The analysis above clearly indicates that the clustering differences between water and alcohols are not seen in the spatial H-bond distribution, which in a way is disappointing. But, we show now that this is not the case for the temporal distributions.

\section{$3.2 \quad$ H-bond life times}

\subsubsection{Water}

Fig.3 shows the H-bond lifetimes distributions $L_{H B}(t)$ for the $\mathrm{SPC} / \mathrm{E}$ water model, in log-scale, for different distances encoded in the color codes of the different curves, and ranging from $r_{c}=2.5 \AA$ to $r_{c}=3.5 \AA$, which cover the distances from under the first peak in Fig. 1 until the first minimum. In terms of the corresponding oxygen-oxygen potential of mean force, these correspond 
to the first neighbour ranges. Fig.3 shows two remarkable features. First, for small distance $r_{c}<3.0 \AA$, one sees a series of peaks, centered around a single maximum which represents the mean bonding lifetime, and these peaks are seen to shift to larger times as $r_{c}$ is increased, as well as decreasing in magnitude. The shift to larger times can be explained as the bonding distance criteria is brought closer to the first maximum of $g(r)$ and beyond, one increases the probability of pair association stability, hence the mean lifetime.

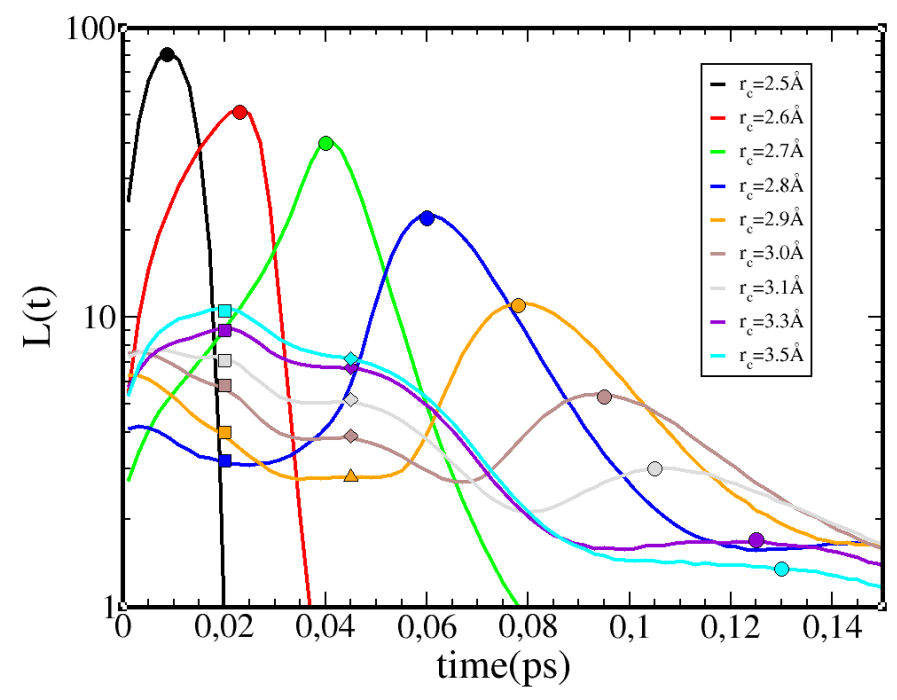

Figure 3: H-bond life time distribution $L(t)$ for SPC/E water, with different H-bonding distances $r_{c}$. The symbols on each curve signal the corresponding peak position (see text): dot for first peak, square for second and triangle for third.

However, as the contact distance is increased, we witness the appearance of 2 secondary peaks, which grow at shorter times than the corresponding first peak lifetime. For example, for the mean H-bonding distance of $r_{c}=2.7 \AA$ (green curve), the main peak is at $40 \mathrm{fs}$, while a broad shoulder at $15 \mathrm{fs}$ witness the growth of the two secondary lifetimes. For the largest H-bonding distance of $r_{c}=3.5 \AA$ (in cyan), the secondary peaks are respectively at $20 \mathrm{fs}$ and $45 \mathrm{fs}$, and their amplitude is far superior to that of the first peak/shoulder at 130fs, since they are respectively of 10 and 7 , almost one order of magnitude larger that the for the first peak. In addition, we see that these two secondary peaks are nearly always at the same respective times of $20 \mathrm{fs}$ and $45 \mathrm{fs}$. Anticipating the corresponding analysis for the alcohols, we infer that these 2 secondary peaks must correspond to lifetimes of bonded pairs within larger clusters, hence are signatures of such clusters. The overall picture which emerges from these findings is that, tightly H-bound molecules ( $\operatorname{small} r_{c}$ ) have high probability but short life times, but less tightly H-bound molecules (larger $r_{c}$ ) are less probable, 
but live longer since they belong to a cluster. But a new problem appears: if the secondary peaks corresponds to H-bonding within a cluster, why two such peaks? Following the same inferring approach, we deduce that two types of clusters are present. From the analysis of the cluster shapes in water, and also in alcohols, we infer that these peaks must correspond respectively to linear and non-linear (globular, or branched) clusters.

Figs.SI-1 and SI-2 of the SI material show that the shape of these life times is very similar across water models. It must therefore be a genuine physical property of real water.

Finally, we note that the conclusions inferred from the analysis above are in contrast to a generic intuitive idea: that clusters could be made of highly and tightly H-bound molecules. The picture which emerges from the present analysis is that larger clusters are less probable than dimers, but they are also live longer. At the opposite range, dimer clusters are highly likely, but they also break faster than larger clusters. Somewhere in between these two extremes, one must have clusters which witness the existence of labile entities, and the 3 peaks of the present finding correspond to typical such clusters.

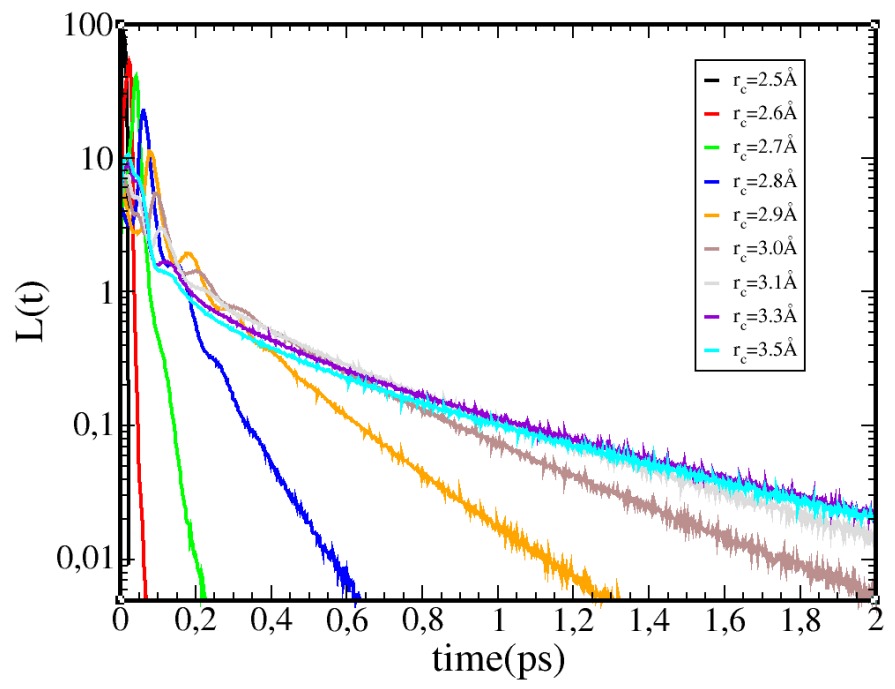

Figure 4: Long time behaviour of the H-bond life time distribution $L(t)$ for the $\mathrm{SPC} / \mathrm{E}$ model.

We now in position to confront our findings with respect to that of Luzar and Chandler [19. Fig.4 shows the long time behaviour of $L(t)$. It is readily seen that, as the H-bonding distance is increased, the fast exponential decay corresponding to smaller H-bonding distances converges towards a slow, and possibly non-exponential behaviour, as witnessed by the merging of the 3 curves corresponding to the range $r_{c}=3.1-3.5 \AA$. Luzar and Chandler have selected $r_{c}=3.5 \AA$ in their entire analysis, and this criterion seems to have been re- 
tained in all subsequent literature [47, 48, 49]. In view of the present analysis, this approach would correspond to a global, almost macroscopical view of the kinetics of association in H-bonded liquids. While this may seem reasonable to make contact with macroscopic physics, we show here that the microscopic physics does not lead to a picture dominated by random distributions, but on the contrary to a selective trinitary view of clustering.

What about other H-bonded liquids? These are usually not studied under the same perspective as water. For example, H-bonding in water is often discussed in terms of flickering clusters 68, or network 69. These specificities do not apply to other H-bonding liquids throughout the literature. Is there a common clustering specificity to all H-bonding liquids?

\subsubsection{Alcohols}

Fig. 5 shows the H-bond lifetimes distributions $L(t)$ for the OPLS methanol model. The comparative analysis for other models is shown in the SI document. A comparison of Fig. 5 with water in Fig. 3 shows immediately that the same global features are present, namely a high first peak which decreases to larger life times for larger $r_{c}$ distances, while intermediate 2 life time peaks emerge at distances around the attractive minimum of the mean force potential.

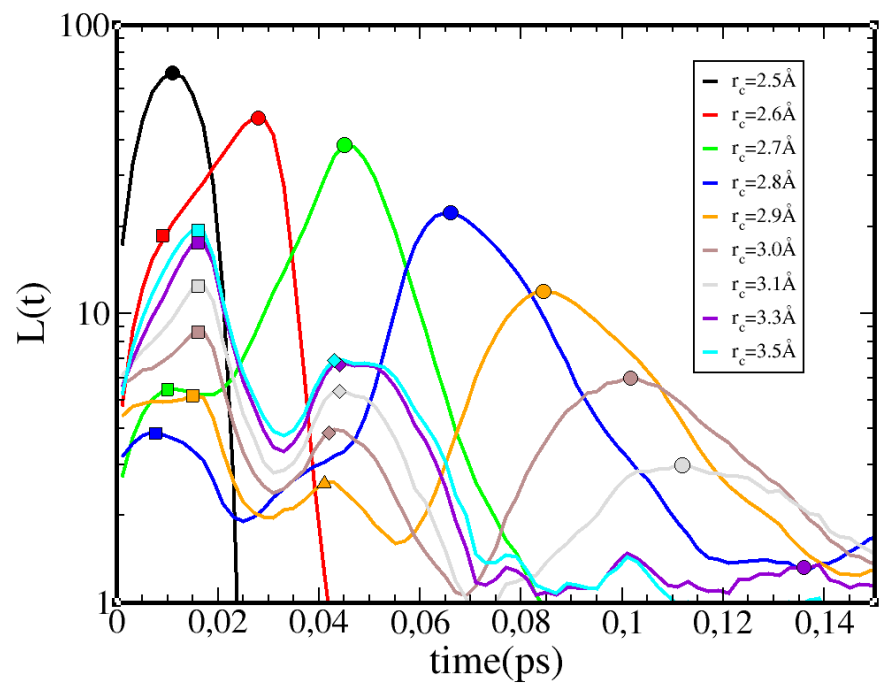

Figure 5: H-bond lifetime distribution for OPLS methanol, with different Hbonding distance $r_{c}$. Symbols and line colors are same as in Fig.3

The principal differences are seen in the values of the maximum amplitudes and positions, which are model dependent to a large extent as one should expect for underlying differences in interactions, but also in the secondary peaks. These latter peaks appear as more marked for methanol than for water, and this is 
more particularly true for the second peak, which is a true peak for the alcohol, while it was more of a shoulder in the case of water. Another similarity with water is the relative insensitivity of the positions of the second and third peaks to the H-bonding $r_{c}$ distance. The fact that the secondary peaks are more marked is in line with the known fact that alcohols form linearly shaped clusters [22, 23, 24, 28] of different topology: chains, loops, lassos [70, 71, 25, 30, 27]. These shapes are not found in water, at least in computer simulation of popular water models. Therefore, the similarity of the peak cannot refer to topological specificity, but to characteristic H-bonding patterns. The cluster analysis of water models shows that water clusters are either globular like or chain like. A similar analysis for the alcohols indicate that linear and branched clusters are mostly present.

Fig.6a and Fig.6b show similar distributions for OPLS ethanol and propanol, as well as Fig.6 of the SI for 1-octanol. Again, the similarity with both methanol and water is striking and enforces the idea that the clustering features of the hydroxyl group are pretty much the same across different $\mathrm{H}$-bonding liquids bearing this group, such as water and mono-ols.
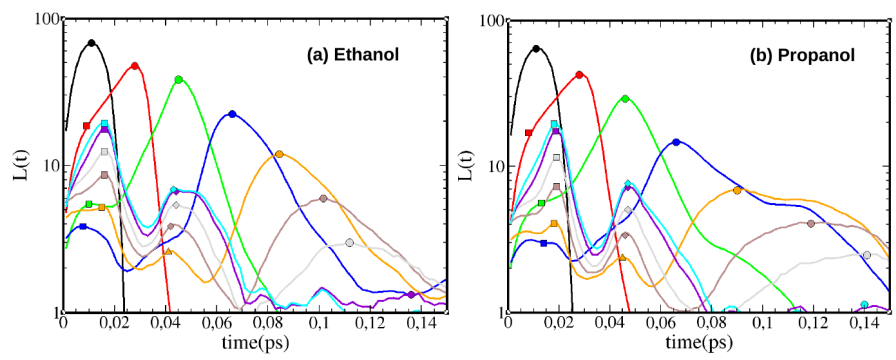

Figure 6: H-bond lifetime distribution for OPLS ethanol (a) and propanol (b), with different $\mathrm{H}$-bonding distance $r_{c}$. Symbols and line colors are same as in Fig.3

The case of 1-propylamine is illustrated in Fig.7 of the SI, and again shows very similar features for $\mathrm{L}(\mathrm{t})$, although this is a very different H-bonding liquid with the amine group NH2, and an acceptor nitrogen atom which is larger than the oxygen atom of water and hydroxyl groups of alcohols. Hence, the distances associated to each curves are different than those in Figs $(3,4)$. This particular liquid confirms that the short time universality for $\mathrm{L}(\mathrm{t})$ uncovered herein is a real feature of H-bonding liquids in general.

Similarly to Fig.4 for water, we compare in Fig.7 the long time decay of $\mathrm{L}(\mathrm{t})$ for several H-bonding liquids, and this for two different values of $r_{c}$. Interestingly, we find that for a given $r_{c}$, all curves tend to lie quite close to one another, and that there is also a species dependance. This is illustrated for $r_{c}=3 \AA$ for water and alcohols. We also note that, since 1-propylamine is a nitrogen based $\mathrm{H}$-bonding liquid, its long time behaviour cannot be compared 


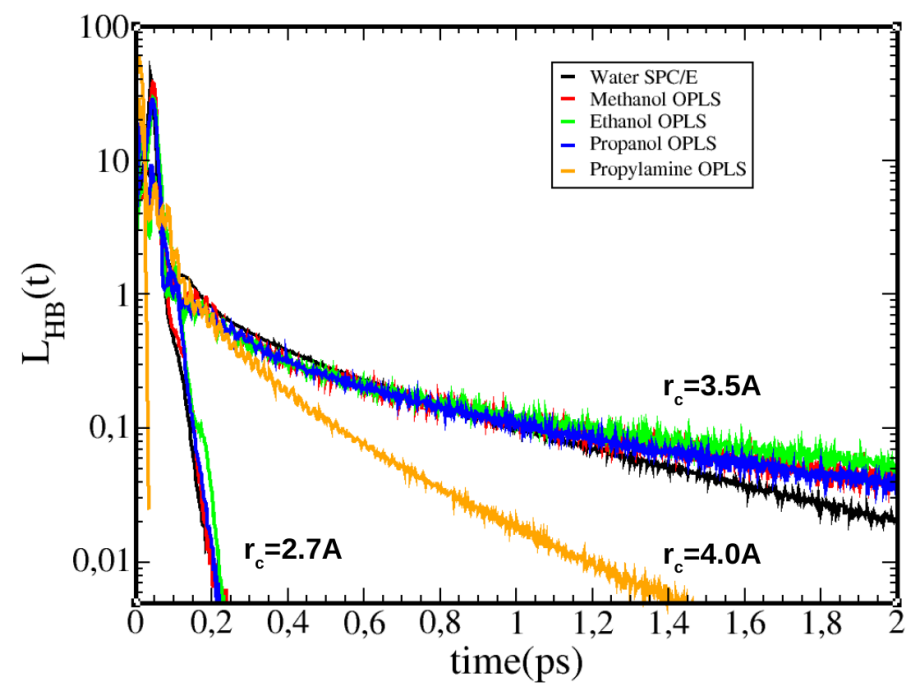

Figure 7: Long time behaviour of the H-bond lifetime distribution for all the models studied herein

with that of oxygen atom based ones. It is interesting to compare the long time analysis of $L(t)$ with the approach of Luzar-Chandler which is based on the analysis of $c(t)$ and the related H-bond kinetics. While the long time behaviour points to differences in Hbonding liquids, the short time transient part, which we study here demonstrates, that the underlying transient dynamics are based on universal elementary cluster structures. This is perhaps the main message of the present findings.

\subsection{A test with the "weak-water" model}

In order to test the present conclusions, and in particular the inference methodology, we have studied previously introduced models of "weak-water" [72]. This model is based on the $\mathrm{SPC} / \mathrm{E}$ water for water, where the partial charges on the oxygen and hydrogen atoms are scaled by a parameter $\lambda(0<\lambda<1)$, allowing to tune the hydrogen bonding from the original model (with $\lambda=1$ ) to a simple Lennard-Jonesium (with $\lambda=0$ ). It was found that from $\lambda \leq 0.6$ the influence of partial charges and hydrogen bonding were not relevant and the model was structurally similar to a simple Lennard-Jones liquid. This model appears here as a useful way to measure the cluster hypothesis for the complex time dependence of $L(t)$. As $\lambda$ is made smaller, the hydrogen bonding abilities decrease, and it is possible to test directly the influence of hydrogen bonding clusters on the shape of $L(t)$.

In the order to preserve the liquid state for small $\lambda$ values and under ambient conditions, it was found necessary to increase the Lennard-Jones energy parameter $\epsilon=\epsilon(\lambda)$ according to the decrease of $\lambda$. In the present test, we have 
bypassed this procedure by doing the test simulations in the NVT Canonical ensemble, hence keeping the volume fixed at that of the real liquid water.

Since the structure of the weak-water liquid is strongly be affected by the decrease of the partial charges, the H-bonding distances must be adjusted appropriately. Fig.8a shows the various $g_{O O}(r)$ for different $\lambda$ values we have used here, namely $\lambda=0.8,0.5$ and 0.2 . The selected bonding distances depend on the position of the maximum, and differ quite a bit from that of the initial SPC/E water, ranging now from $2.7 \AA$ to $4.5 \AA$. It is important to note that, while we vary the bonding distance criteria, we keep the angular criterion the same as that for pure water, which is that the angle O-H-O is $180 \pm 30$. This means that, even though weaker water model may have different bonding angles, we will still select only a sub class of those obeying tetrahedral bonding directions. This is justified by the fact that these models do not have as strong $\mathrm{H}$-bonding tendencies as $\mathrm{SPC} / \mathrm{E}$ water, hence the angular bias is less significant than the distance of bonding. But we will need to take into this bias in order to interpret the data.
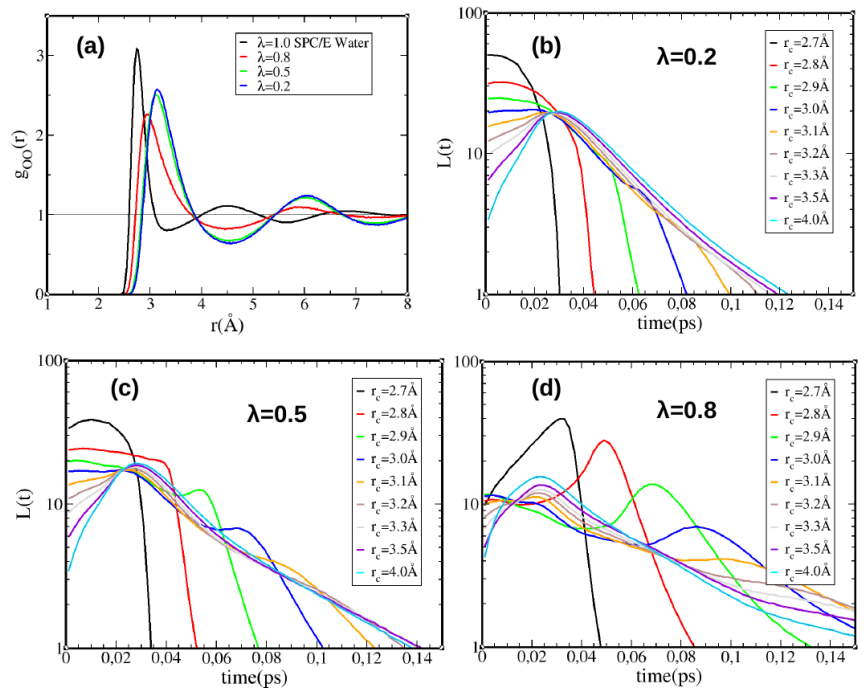

Figure 8: Weak water models data. (a) Comparison of the RDF of various models; (b) H-bond lifetimes for $\lambda=0.2$; (c); (b) H-bond lifetimes for $\lambda=0.5$; (d) H-bond lifetimes for $\lambda=0.8$.

Fig.8b shows the H-bond lifetimes for $\lambda=0.2$, which is very close to a Lennard-Jones system. For very small bonding distances $r_{c} \leq 3.0 \AA$, we note that the $L(t)$ do not exhibit a clear maximum, and sometimes even show a plateau-like behaviour, such as for $R=3.0 \AA$ (blue curve). This is a direct consequence of the angular bias described above. However, from bonding distance $r_{c} \geq 3.1 \AA$, we observe a clear maximum, and this maximum is nearly the same for all subsequent $r_{c}$ values. This is what we would expect in a quasi-LJ system, 
where pairing is nearly isotropic. We also note that this lifetime distribution is not necessarily about isolated pairs, and could involve those in larger clusters. We note the presence of intriguing small shoulder-like features at large times, such as for the green, blue and yellow curves, but these features cannot be interpreted from Fig.8b alone, but will become clearer from the analysis of the next cases.

Fig.8c shows the H-bond lifetimes for $\lambda=0.5$, which has the same pair distribution as the $\lambda=0.2$ case, as seen in Fig.8a. We note that most of the features observed previously equally appear here - supporting the structural analogy, but the previously noted intriguing shoulder structures has now grown into peak structures and are very apparent. However, it still remain difficult to interpret them by simple inference.

Fig.8d is for the case $\lambda=0.8$, which is the closest to SPC/E water, and should be compared with Fig.3. The previous intriguing peaks have now grown to invade the entire figure, and, by comparison with Fig.3, represent the dominant contribution to the H-bond lifetimes for each $\mathrm{R} r_{c}$ value, as we have interpreted them when discussing Fig.3. But now, we can finally understand the smaller features in Fig.8c, namely the origin of the secondary peaks, precisely because these were the dominant features in the previous Figs.8b-c. Indeed, we have interpreted them as peaks related to bonded pairs within clusters. This is precisely the conclusion we have reached when discussing Figs.3-6, but by inference. To summarize, the study of the weak-water models allow us to confirm that the secondary peak features are indeed related to clusters.

\subsection{Influence of the alkyl tails}

Although the present study reveals similarities in the H-bonding life times, one would expect that the presence of non-bonding alkyl tails would affect the lifetime. This is illustrated in Fig.9 for all the liquids studied in this work, water, methanol to 1-octanol and 1-propylamine. Since the alkyl tail influence is best seen at largest distance cutoff we have considered $r_{c}=3.5 \AA$ for all systems except for propylamine, for which we have used appropriately $r_{c}=4.0 \AA$.

For clarity, each curve has been shifted by $\log (10)$ from the previous one. The bottom black curve is for water, and serves only as a reference, since water has no alkyl tail, and consequently does not show anything particular other than the features discussed above. However, all the alcohol curves show tiny oscillations past the 3rd peak. Higher alcohols such as 1-propanol and 1-octanol have even their 3rd peak weakly modulated. We attribute these oscillations in lifetime to the presence of the alkyl tail "bath", which surrounds the $\mathrm{OH}$ clusters, and affects the decay of their life time. This interesting feature further supports the interpretation that the 3rd peak is associated with the evolution of the cluster topology in time. This feature is naturally absent from water because there are no alkyl groups. However, the case of the propylamine (the upper most curve in purple) is very interesting. In previous studies [4, we have compared the clustering of neat 1propanol to that of 1-propylamine, and shown that the clustering of the amine group was not so important, both in size and 


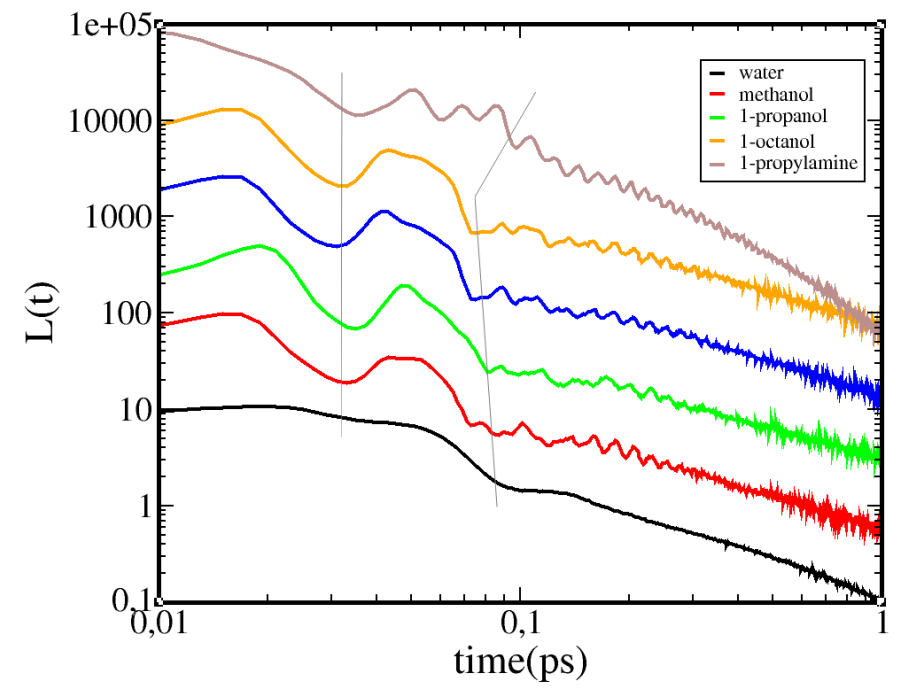

Figure 9: Influence of alkyl tails on lifetime distribution for methanol (red), ethanol (green), propanol(blue), octanol (gold) and propylamine (purple). The black curve is for water and serves as a reference. The grey lines serve to delimitate the 2 nd and 3 rd peaks regions mentioned in the text.

shape, as the chain patterns observed for 1propanol. The alkyl chain, being the same between the 2 species, plays an indirect role in this incomplete clustering of the NH2 amine groups. What we observe in Fig.9 is that the role of the alkyl tail is so important that it "bites" into the 3rd peak which is about cluster topology, and makes even this peak look less characteristic compared to those of the alcohols. These oscillations also last longer than in the case of alcohols. This figure reveals the dynamical role played by the seemingly neutral alkyl tail background. The role of these tails was emphasized in a previous study [32] of the shape of the Xray pre-peak feature of alcohols. This role is confirmed through the present study.

\section{Discussion and Conclusion}

While chemical matter, and more importantly bio-chemical matter, is known to be made of labile objects, in addition to well defined atoms and molecules. It raises the question whether such fleeting objects could play a role as important as the permanent ones. The pertinence of this question is enforced by the recognition that liquid assemblies of atoms and molecules are subject to fluctuations which depend on the nature of the interactions, and in particular highly directional ones such as the H-bond interaction. Self assembly is the driving mechanism of many complex systems, such as in soft-matter and biological systems. The microscopic interaction at the root of self-assembly is the H-bonding 
process. This mechanism can be relatively well captured by classical force field simulation using Coulomb charge pairing.

The calculations reported herein show that, for a given H-bonding distance $r_{c}$, the H-bond lifetime is essentially dominated by one mean lifetime for distances $r_{c}$ smaller than the first peak of $g(r)$, but that two secondary peaks appear for larger distances until the first minimum of $g(r)$, and that these become dominant as $r_{c}$ is increased. We have attributed these peaks to linear and non-linear cluster geometries. In addition, the present study reveals a previously unexpected similarity in H-bonding lifetime distribution in the small distance and short time regime corresponding to distances under $3.5 \AA$ and under $150 \mathrm{fs}$, and this independently of the nature of the H-bonding group. We have provided convincing empirical arguments for the existence of three type of H-bond based clustering specificities in three different associated liquids, water, mono-ols and amines. These arguments suggest that there exist two distinct families of clusters, inside which the dimer lifetime plays a very different role. The first family concerns linearly shaped clusters, whether these are line-like or circular. The second family, we have refered as non-linear clusters, which covers all other forms, such as globular -as those found in water, or branched, such as lasso-shaped found in mono-ols [73, 32].

The calculations concern primarily lifetime of pair of H-bonded particles. But such pairs often reside inside specific clusters, hence their lifetime is affected by the life of the entire cluster. Because of the cooperative motions of particles linked within a cluster, we expect that the these motions affect the lifetime statistics in particular ways, as to emerge the 3 peaks observed in the data reported herein.

\section{Acknowledgments}

This work has been supported in part by the Croatian Science Foundation under the project UIP-2017-05-1863 "Dynamics in micro-segregated systems".

\section{References}

[1] A. J. Goshe, I. M. Steele, C. Ceccarelli, A. L. Rheingold and B. Bosnich, Proceedings of the National Academy of Sciences, 2002, 99, 4823-4829

[2] F. Paul, C. Wehmeyer, E. T. Abualrous, H. Wu, M. D. Crabtree, J. Schöneberg, J. Clarke, C. Freund, T. R. Weikl and F. Noé, Nature Communications, 2017, 8, 1095

[3] O. Glatter, G. Fritz, H. Lindner, J. Brunner-Popela, R. Mittelbach, R. Strey and S. U. Egelhaaf, Langmuir, 2000, 16, 8692-8701

[4] S. De, V. K. Aswal, P. S. Goyal and S. Bhattacharya, The Journal of Physical Chemistry B, 1998, 102, 6152-6160 
[5] J. Berghausen, J. Zipfel, P. Lindner and W. Richtering, The Journal of Physical Chemistry B, 2001, 105, 11081-11088

[6] D. C. Augenstein, K. Thrasher, A. J. Sinskey and D. I. C. Wang, Biotechnology and Bioengineering, 1974, 16, 1433-1447

[7] H. Spahn-Langguth and L. Z. Benet, Microsomal Acyl Glucuronidation: Enzyme-Kinetic Studies with Labile Glucuronides, 1999

[8] Y. Kim, J. Park and M.-J. Kim, ChemCatChem, 2011, 3, 271-277

[9] J. T. Trevors and R. Psenner, FEMS Microbiology Reviews, 2001, 25, 573582

[10] D. Deamer, S. Singaram, S. Rajamani, V. Kompanichenko and S. Guggenheim, Philosophical transactions of the Royal Society of London. Series B, Biological sciences, 2006, 361, 1809-1818

[11] L. Almásy, A. Kuklin, M. Požar, A. Baptista and A. Perera, Phys. Chem. Chem. Phys., 2019, 21, 9317-9325

[12] I. Jukić, M. Požar and B. Lovrinčević, Phys. Chem. Chem. Phys., 2020, 22, 23856-23868

[13] R. F. Lake and H. Thompson, Proceedings of the Royal Society of London. Series A. Mathematical and Physical Sciences, 1966, 291, 469-477

[14] A. S. N. Murthy and C. N. R. Rao, Applied Spectroscopy Reviews, 1968, 2, 69-191

[15] S. Craven and F. Bentley, Appl. Spectrosc., 1972, 26, 449-453

[16] S. Craven and F. Bentley, Appl. Spectrosc., 1972, 26, 242-247

[17] W. L. Jorgensen, Journal of the American Chemical Society, 1981, 103, $341-345$

[18] F. W. Starr, J. K. Nielsen and H. E. Stanley, Phys. Rev. E, 2000, 62, $579-587$

[19] A. Luzar and D. Chandler, Nature, 1996, 379, 55-57

[20] W. Pierce and D. MacMillan, Journal of the American Chemical Society, 1938, 60, 779-783

[21] B. Warren, Phys. Rev., 1933, 44, 969-973

[22] M. Magini, G. Paschina and G. Piccaluga, The Journal of Chemical Physics, 1982, 77, 2051-2056

[23] A. Narten and A. Habenschuss, The Journal of Chemical Physics, 1984, 80, 3387-3391 
[24] K. S. Vahvaselkä, R. Serimaa and M. Torkkeli, Journal of Applied Crystallography, 1995, 28, 189-195

[25] A. Karmakar, P. Krishna and R. Joarder, Physics Letters A, 1999, 253, $207-210$

[26] T. Yamaguchi, K. Hidaka and A. Soper, Molecular Physics, 1999, 96, 1159

[27] C. Benmore and Y. Loh, The Journal of Chemical Physics, 2000, 112, $5877-5883$

[28] M. Tomšič, A. Jamnik, G. Fritz-Popovski, O. Glatter and L. Vlček, The Journal of Physical Chemistry B, 2007, 111, 1738-1751

[29] A. Sahoo, S. Sarkar, V. Bhagat and R. N. Joarder, The Journal of Physical Chemistry A, 2009, 113, 5160-5162

[30] A. Vrhovšek, O. Gereben, A. Jamnik and L. Pusztai, The Journal of Physical Chemistry B, 2011, 115, 13473-13488

[31] J. Cerar, A. Lajovic, A. Jamnik and M. Tomšič, Journal of Molecular Liquids, 2017, 229, 346 - 357

[32] M. Požar, J. Bolle, C. Sternemann and A. Perera, The Journal of Physical Chemistry B, 2020, 124, 8358-8371

[33] F. C. Hagemeister, C. J. Gruenloh and T. S. Zwier, The Journal of Physical Chemistry A, 1998, 102, 82-94

[34] K. M. Murdoch, T. D. Ferris, J. C. Wright and T. C. Farrar, The Journal of Chemical Physics, 2002, 116, 5717-5724

[35] W. Wrzeszcz, M. Tomza, P.and Kwaśniewicz, S. Mazurek, R. Szostak and M. Czarnecki, RSC Advances, 2016, 6, 37195

[36] V. Pogorelov, C. Y., Y. Vaskivskyi, L. G. Pettersson, I. Doroshenko, V. Sablinskas, V. Balevicius, J. Ceponkus, K. Kovaleva, A. Malevich and G. Pitsevich, Journal of Molecular Liquids, 2016, 216, 53 - 58

[37] M. P. Balanay, D. H. Kim and H. Fan, The Journal of Chemical Physics, 2016, 144, 154302

[38] T. Kosztolányi, I. Bakó and G. Pálinkás, The Journal of Chemical Physics, 2003, 118, 4546-4555

[39] R. Ludwig, ChemPhysChem, 2005, 6, 1369-1375

[40] L. Zoranić, F. Sokolić and A. Perera, The Journal of Chemical Physics, 2007, 127, 024502

[41] A. Perera, F. Sokolić and L. Zoranić, Physical Review E, 2007, 75, 060502(R) 
[42] J. Lehtola, M. Hakala and K. Hämäläinen, The Journal of Physical Chemistry $B, 2010, \mathbf{1 1 4}, 6426-6436$

[43] M. Požar, B. Lovrinčević, L. Zoranić, M. Mijaković, F. Sokolić and A. Perera, The Journal of Chemical Physics, 2016, 145, 064509

[44] M. Požar and A. Perera, Journal of Molecular Liquids, 2017, 227, 210

[45] G. Schreiber, G. Haran and H.-X. Zhou, Chemical reviews, 2009, 109, 839860

[46] M. Haughney, M. Ferrario and I. R. McDonald, The Journal of Physical Chemistry, 1987, 91, 4934-4940

[47] E. Guardia, J. Martí, J. Padró, L. Saiz and A. Komolkin, Journal of Molecular Liquids, 2002, 96-97, 3 - 17

[48] I. Skarmoutsos and E. Guardia, The Journal of Physical Chemistry B, 2009, 113, $8898-8910$

[49] J. Cerar, A. Jamnik, I. Pethes, L. Temleitner, L. Pusztai and M. Tomšič, Journal of Colloid and Interface Science, 2020, 560, 730 - 742

[50] A. Luzar, The Journal of Chemical Physics, 2000, 113, 10663-10675

[51] H. F. M. C. Martiniano and N. Galamba, The Journal of Physical Chemistry $B, 2013, \mathbf{1 1 7}, 16188-16195$

[52] V. P. Voloshin and Y. I. Naberukhin, Journal of Structural Chemistry, $2009, \mathbf{5 0}, 78-89$

[53] A. Geiger, P. Mausbach, J. Schnitker, R. Blumberg and S. H., Journal de Physique Colloques C, 1984, 45, C7-13-C7-30

[54] H. J. C. Berendsen, J. R. Grigera and T. P. Straatsma, The Journal of Physical Chemistry, 1987, 91, 6269-6271

[55] J. L. F. Abascal and C. Vega, The Journal of Chemical Physics, 2005, 123, 234505

[56] W. Jorgensen, The Journal of Physical Chemistry, 1986, 90, 1276

[57] B. Chen, J. Potoff and J. Siepmann, The Journal of Physical Chemistry B, 2001, 105, 3093

[58] S. Pronk, S. Páll, R. Schulz, P. Larsson, P. Bjelkmar, R. Apostolov, M. Shirts, J. Smith, P. Kasson, D. van der Spoel, B. Hess and E. Lindahl, Bioinformatics, 2013, 29, 845

[59] M. Abraham, T. Murtola, R. Schulz, S. Páll, J. Smith, B. Hess and E. Lindahl, SoftwareX, 2015, 1-2, 19 
[60] J. Martínez and L. Martínez, Journal of Computational Chemistry, 2003, $\mathbf{2 4}, 819$

[61] R. Hockney, in Methods in computational physics, vol. 9, ed. B. Alder, S. Fernbach and M. Rotenberg, Orlando Academic Press, 1970, vol. 9, ch. The potential calculation and some applications, pp. 135-221

[62] T. Darden, D. York and L. Pedersen, The Journal of Chemical Physics, 1993, 98, 10089

[63] B. Hess, H. Bekker, H. Berendsen and J. Fraaije, Journal of Computational Chemistry, 1997, 18, 1463

[64] S. Nose, Molecular Physics, 1984, 52, 255

[65] W. Hoover, Physical Review A, 1985, 31, 1695

[66] M. Parrinello and A. Rahman, Physical Review Letters, 1980, 45, 1196

[67] M. Parrinello and A. Rahman, Journal of Applied Physics, 1981, 52, 7182

[68] H. S. Frank and W.-Y. Wen, Discuss. Faraday Soc., 1957, 24, 133-140

[69] J. Perram and S. Levine, null, 1971, 21, 701-708

[70] S. Sarkar and R. N. Joarder, The Journal of Chemical Physics, 1993, 99, 2032-2039

[71] S. Sarkar and R. N. Joarder, The Journal of Chemical Physics, 1994, 100, $5118-5122$

[72] B. Kežić, R. Mazighi and A. Perera, Physica A: Statistical Mechanics and its Applications, 2013, 392, 567-582

[73] J.-H. Guo, Y. Luo, A. Augustsson, S. Kashtanov, J.-E. Rubensson, D. K. Shuh, H. Ågren and J. Nordgren, Phys. Rev. Lett., 2003, 91, 157401 